\title{
Swallow Syncope After Whiplash Neck Injury
}

\author{
Luka Lipar, MD, PhD, ${ }^{1}$ Pia Vovk, MD,${ }^{2}$ Tereza Weiss, $M D,{ }^{3}$ Jus Ksela, $M D, \mathrm{PhD}^{4}$ \\ Departments of ${ }^{1}$ Cardiology, ${ }^{2}$ Anaesthesiology and Surgical Intensive Therapy, and ${ }^{4}$ Cardiovascular Surgery, University Medical \\ Center, Ljubljana, Ljubljana, Slovenia; ${ }^{3}$ Medical School, University of Ljubljana, Ljubljana, Slovenia
}

\section{ABSTRACT}

Swallow syncope is a rare dysautonomic syndrome characterized by temporary loss of consciousness upon swallowing solid foods or liquids, displaying primarily in individuals with an underlying structural or functional esophageal or cardiac pathology. However, the evidence also suggests that isolated vagal dysfunction or abnormal reactions of esophageal mechanoreceptors after mechanical irritation, demyelination, or trauma can potentially evoke a cardioinhibitory response or vasodepression upon swallowing. We present a case of a 49-year-old otherwise healthy female patient who developed swallow syncope two weeks after whiplash neck injury acquired in a rear-end collision. After no evident anatomical and/or functional cardiac or esophageal pathology was diagnosed using several diagnostic procedures, the final diagnosis was confirmed by a provocative tilt-table test while ingesting solid food. Subsequently, a dual-chamber pacemaker was implanted, relieving the patient from troublesome symptoms. Abnormal reactions of esophageal mechanoreceptors to stimuli associated with food ingestion and/or dysfunction of afferent and efferent vagal fibers due to stretch injury and related neck trauma (acquired during the car accident) may be the leading pathophysiological mechanisms of swallow syncope in our patient.

\section{INTRODUCTION}

Swallow syncope is a rare clinical entity, belonging to the category of reflex-mediated vasomotor inhibitory syndromes [Kang 2005; Omi 2006; Mitra 2011]. It is characterized by temporary loss of consciousness during or shortly after swallowing solid foods or liquids. The vast majority of affected patients have an underlying structural or functional esophageal or cardiac pathology. However, several reports have also suggested that isolated aberrant conduction through afferent and efferent vagal fibers and abnormal reactions of esophageal mechanoreceptors caused by mechanical irritation, demyelination, or trauma can potentially evoke a cardioinhibitory response or vasodilatation of vascular bed upon swallowing in certain individuals [Kang 2005; Mitra 2011]. Here, we present a case of swallow syncope after whiplash trauma

Received November 14, 2017; accepted fanuary 17, 2018.

Correspondence: Fus Ksela, MD, PhD, Department of Cardiovascular Surgery, University Medical Center Ljubljana, Zaloska cesta 7, 1000 Ljubljana, Slovenia; +386-1-522-8251 (e-mail: jus.ksela@kcl.si). in the absence of clinically evident functional and structural esophageal or cardiac pathology in an adult female patient.

\section{CASE REPORT}

A 49-year-old healthy Caucasian female with no relevant medical history suffered a whiplash injury to her neck during a massive motor vehicle collision. After admission to the emergency department, she complained of severe neck pain, and physical examination showed limited neck motion with decreased skin sensation and muscular weakness in the upper extremities. An X-ray and a computed tomography (CT) scan of the cervical spine performed at that time revealed a loss of normal neck lordosis with no bone fractures or evident soft tissue injuries. Thus, the patient was diagnosed with a grade 3 whiplash injury, WAD III, according to Quebec Task Force Clinical Classification of Whiplash Associated Disorders (WAD) [Spitzer 1995] and treated conservatively, first with soft collar immobilization followed by a rehabilitation program, including physical therapy exercises and postural modifications. However, with no prior history of fainting spells, she suddenly began to suffer from episodes of lightheadedness and syncope two weeks following her whiplash injury. After numerous episodes and several hospital admissions in the subsequent months, she started to notice a connection between losing consciousness and ingesting solid foods, especially nuts, raw carrots and steaks.

During the course of her illness, ECGs, Holter monitoring, magnetic resonance imaging (MRIs) of the head, neck and thorax, standard tilt table test, echocardiogram, esophagogastroduodenoscopy, electroencephalography (EEG) and carotid ultrasound imaging were performed on an out-patient basis, showing no specific structural or evident functional abnormalities. After admission to our institution, a provocative tilt table test while ingesting solid food was performed, revealing an intermittent second- and third-degree atrioventricular $(\mathrm{AV})$ block with prolonged asystole during swallowing, accompanied by severe hypotension and near-loss of consciousness (Figure 1). Moments after finishing swallowing, the AV block resolved and consequently her blood pressure normalized and her symptoms diminished. Additionally, neither the Valsalva maneuver nor carotid massage performed during and after tilt-testing could produce bradycardia, AV block or syncope. Next, with patient encouraged to ingest provocative foods, Holter monitoring was repeated, this time showing an intermittent third degree AV block induced by swallowing the food. Sinoatrial (SA) and AV node function, subsequently evaluated by an atropine test was normal. A 


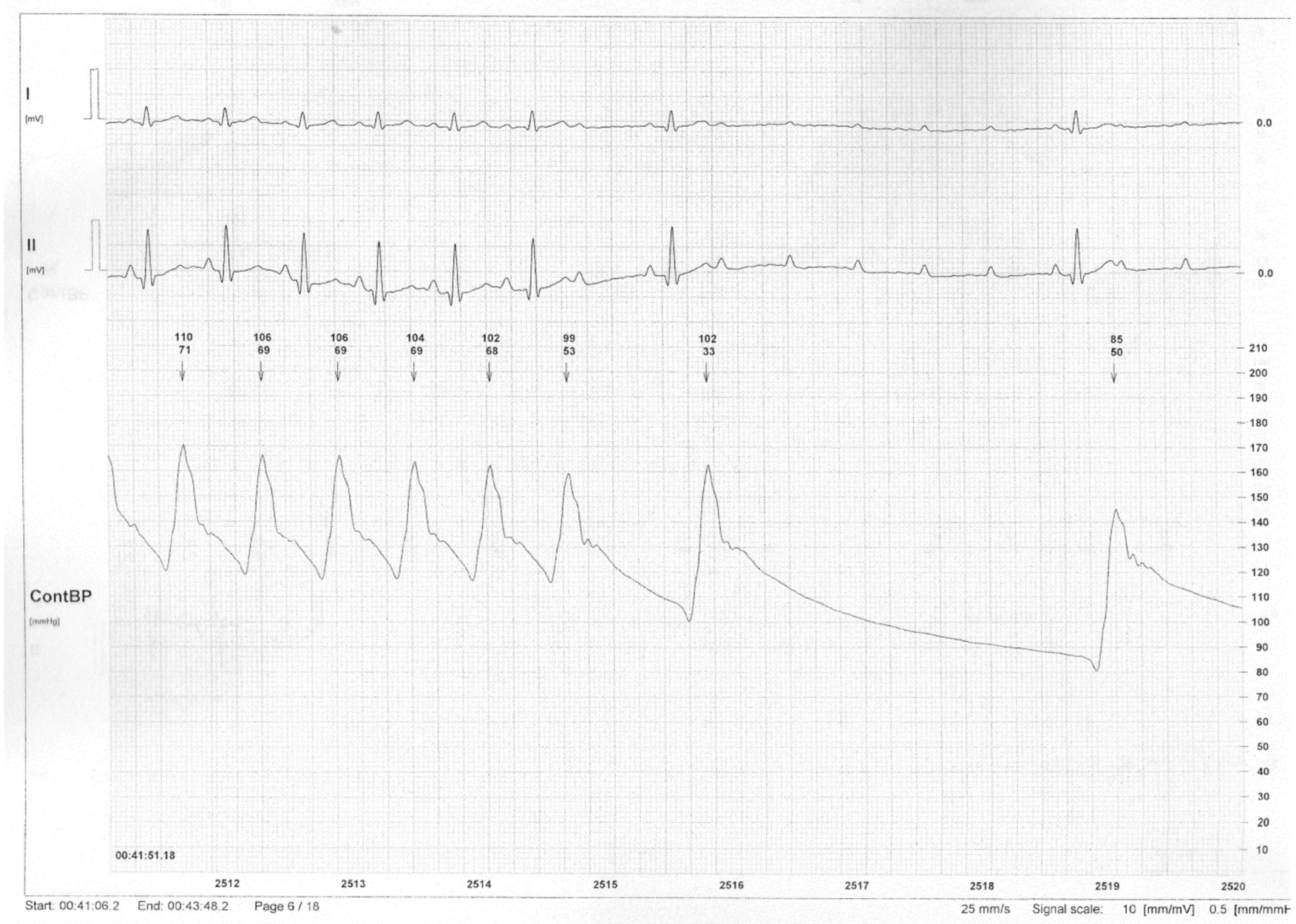

Figure 1. Second degree AV block with 6:1 conduction and concomitant drop in blood pressure during ingestion of solid food as recorded during tilt-testing. The upper two tracings represent two ECG channels; the lower tracing represent continuous blood pressure measurement (ContBP).

diagnosis of swallow syncope was thus confirmed and consequently, a dual-chamber pacemaker implanted in a standardized surgical fashion. The surgical procedure and the early postoperative course were uneventful and the patient was discharged on the first postoperative day. One year after the procedure she is clinically stable, with no recurrent episodes of lightheadedness or syncope even upon ingestion of solid food, with improved quality of life and managed on the outpatient basis.

\section{DISCUSSION}

Swallow or deglutition syncope is an extremely rare clinical disorder, etiologically belonging to the group of reflexmediated vasomotor inhibitory syndromes, similar to defecation, micturition or cough syncope [Carey 1999; Garg 2013]. In swallow syncope, loss of consciousness occurs during or shortly after swallowing solid foods, liquids, or both due to reduced blood flow to the brain, induced by intermittent arrhythmias and/or vasodepression with vasodilation in the absence of cardiac inhibition [Kang 2005; Mitra 2011]. Very hard or sticky solid foods, carbonated beverages, and ice-cold liquids are the most commonly reported ingestion triggers in the existing literature. Among different cardiac rhythm disturbances encountered in affected individuals, intermittent $\mathrm{AV}$ conduction blocks are most commonly reported. However, sinus bradycardia, sinus arrest, atrial and ventricular asystole, or rarely, tachyarrhythmias have also been described [Kang 2005; Mitra 2011]. The syndrome is known to occur in patients with structural or functional disorders of the esophagus or in various settings of cardiac pathologies, including: esophageal spasm, stricture, achalasia, diverticula, cancer, hiatal hernia, inferior wall myocardial infarction, rheumatic myocarditis, aortic valve calcification interrupting the His bundle, and with different heart disease medications. Noteworthy, in a considerable number of cases, no underlying disease causing loss of consciousness upon food ingestion can be found, as was the case with our patient [Kang 2005; Mitra 2011].

Several pathophysiological mechanisms behind swallow syncope have been proposed, however, a vagally-mediated reflex with excessive parasympathetic output to the heart, with 
or without concomitant sympathetic withdrawal, seems to play a central role in this phenomenon [Kang 2005; Omi 2006, Mitra 2011]. The esophagus and the heart share a common innervation via the vagus nerve. Normally, mechanoreceptors in the esophagus sense distension upon swallowing food or liquids and subsequently send afferent impulses along the esophageal plexus to the nucleus solitarius tract at the brainstem. The efferent impulses from the brainstem reach the SA and $\mathrm{AV}$ node via the right and left vagus nerve, respectively [Kang 2005; Mitra 2011]. The structural and functional esophageal and cardiac disorders mentioned above are likely to induce improper function of each part of the autonomic neural loop (the afferent and efferent vagal fibers and esophageal receptors), thereby provoking swallow syncope. However, in absence of an underlying pathology, an isolated dysfunction of mechanoreceptors or vagal fibers due to mechanical irritation, demyelination or trauma, induced by various triggers such as illuminating gas inhalation, diphtheria infection, or surgical trauma, was suggested as the primary pathophysiological mechanism by several authors [Carey 1999; Kang 2005; Garg 2013].

Whiplash neck injury is characterized by severe stretch of the soft tissues of the neck anterior to the cervical spine during the hyperextension phase of the whiplash trauma [Davis 2000; Uhrenholt 2011], and is routinely classified according to the Quebec Task Force Clinical Classification, staging patients in 5 WAD grades based on the severity of signs and symptoms following whiplash (neck pain, musculoskeletal deficits, neurological deficits, and bone fractures/dislocations) [Spitzer 1995]. Although the majority of whiplash injuries demonstrate little or no evidence of severe tissue damage with diagnostic imaging, the posttraumatic tissue edema, small-scale hematoma, and microtrauma of target structures may reflect in severe dysfunction of vital neck organs, such as the esophagus, trachea, carotid artery, jugular vein and neural system [Brademann 1998; Davis 2000; Omar 2007; Uhrenholt 2011]. In our otherwise healthy patient, with no prior history of fainting spells, the alteration, or loss of consciousness upon swallowing solid foods first appeared two weeks after the whiplash injury, which strongly suggested that stretchinduced injury of mechanoreceptors and vagal fibers acquired during car accident related neck trauma might be the fundamental pathophysiological mechanism in this clinical case.

The spectrum of symptoms in swallow syncope is highly variable in both frequency and intensity, making the diagnostic procedure difficult and usually long lasting [Kang 2005; Omi 2006; Mitra 2011]. A definitive diagnosis requires careful history taking to recognize a clear temporal relationship between swallowing liquids or solid foods and lightheadedness and/or syncope. Provocative testing with various types of liquid and solid foods should be performed and underlying structural and functional esophageal or cardiac pathologies ruled out [Mitra 2011; Garg 2013]. With unexplained episodes of syncope, especially when there is an association between symptoms and food ingestion, the clinician should think of swallow syncope [Garg 2013].

Management of swallow syncope includes discontinuation of all medications causing bradycardia and $\mathrm{AV}$ conduction block. If there is a known nutritional trigger, avoidance of the specific foods or liquids and behavioral modification to change eating habits might alleviate the symptoms in patients with infrequent episodes of syncope [Kang 2005; Mitra 2011; Garg 2013]. To prevent bradyarrhythmias, numerous anti-cholinergic medications like atropine, scopolamine, and propantheline have been suggested [Mitra 2011]. Sympathomimetic agents like adrenaline and isoprenaline have been used to increase the ventricular rate directly; however, these drugs can be poorly tolerated [Carey 1999; Kang 2005; Omi 2006; Garg 2013]. Correction of any underlying esophageal or cardiac pathology should be undertaken if present [Mitra 2011]. In individuals with a predominantly bradycardiainduced syncope, permanent pacemaker implantation usually represents the most successful treatment, relieving symptoms and improving the patient's quality of life, as in our case [Carey 1999; Kang 2005; Omi 2006; Mitra 2011; Garg 2013].

\section{CONCLUSION}

In conclusion, we present, to our knowledge, a so far unpublished clinical scenario of swallow syncope after whiplash neck injury. In the absence of clinically evident functional and structural esophageal or cardiac pathology, abnormal posttraumatic reactions of esophageal mechanoreceptors to stimuli associated with food ingestion and/or dysfunction of afferent and efferent vagal fibers due to microtrauma, a small scale hematoma, or edema acquired during a car accident related neck injury may be, in our opinion, the leading pathophysiological mechanisms of swallow syncope in this patient.

\section{REFERENCES}

Brademann G, Reker U. 1998. Paralysis of the superior laryngeal nerve after whiplash trauma. Laryngorhinootologie 77:3-6.

Carey BJ, de Caestecker J, Panerai RB. 1999. More on deglutition syncope. N Engl J Med 341:1316-17.

Davis CG. 2000. Injury threshold: whiplash - associated disorders. J Manipulative Physiol Ther 23:420-7.

Garg S, Girotra M, Glasser S, Dutta SK. 2013. Swallow syncope: clinical presentation, diagnostic criteria, and therapeutic options. Saudi J Gastroenterol 20:207-11.

Kang KH, Cho WH, Kim MC, et al. 2005. Cases of swallow syncope induced by the activation of mechanoreceptors in the lower esophagus. Korean J Intern Med 20:68-71.

Mitra S, Ludka T, Rezkalla SH, Sharma PP, Lou J. 2011. Swallow syncope: a case report and review of the literature. Clin Med Res 9:125-9.

Omar N, Alvi F, Srinivasan MS. 2007. An unusual presentation of whiplash injury: long thoracic and spinal accessory nerve injury. Eur Spine J 16:275-7.

Omi W, Murata Y, Yaegashi T, et al. 2006. Swallow syncope, a case report and review of the literature. Cardiology 105:75-9.

Spitzer WO, Skovron ML, Salmi LR, et al. 1995. Scientific monograph of the Quebec task force on whiplash associated disorders: redefining whiplash ant its management. Spine 20:8S-58S.

Uhrenholt L, Freemann MD, Jurik AG, et al. 2011. Esophageal injury in fatal rear-impact collisions. Forensic Science International 206:e52-e57. 